Die weihnächtliche Schlemmerzeit ist vorbei, nun kommt die Zeit der guten Vorsätze - vielleicht hilft die Lektüre des neu erschienenen 6. Schweizerischen Ernährungsberichtes des Bundes dabei. Auch in der Schweiz steigt die Rate der Übergewichtigen, mit den allseits bekannten Folgen für die Gesundheit der Betroffenen. Warum fällt es uns so schwer, die allgemein bekannten Regeln eines gesunden Essverhaltens umzusetzen? Blicken wir zurück: Während ein paar hunderttausend Jahren hat es entscheidend zu unserem Überleben beigetragen, dass wir viel gegessen haben - wenn Essen vorhanden war - und Fett ansammeln konnten als Reserven für magere Zeiten. Wir mussten uns viel bewegen, Nahrungsmittelbeschaffung war ein anstrengendes Unterfangen! Für üppige Zeiten und volle Einkaufsregale sind wir nicht geschaffen: Es braucht gemeinsame Anstrengungen verschiedener Akteure, damit unser Jäger- und-Sammlerinnen-Körper in Zeiten von Nahrungsmittelüberfluss und Bewegungsmangel nicht aus den Fugen gerät.

\title{
Sechster Schweizerischer Ernährungsbericht und Schweizer Ernährungsstrategie 2013-2016
}

\author{
Andrea Renggli, Michael Beer, \\ Pascale Mühlemann, \\ Nadine Stoffel-Kurt
}

Korrespondenz:

Dr. Andrea Renggli

Bundesamt für Gesundheit Abt. Lebensmittelsicherheit Sektion Ernährungs- und Toxikologische Risiken Stauffacherstrasse 101 CH-8004 Zürich

andrea.renggli[at]bag.admin.ch
Die Ernährung hat einen wesentlichen Einfluss auf die Gesundheit: Eine ausgewogene Ernährung deckt nicht nur den Bedarf an Energie (Kalorien) und Nährstoffen, verhindert eine überschüssige Energiezufuhr und beeinflusst das körperliche und geistige Wohlbefinden positiv, sondern sie trägt auch dazu bei, die Gesundheit zu erhalten und ernährungsabhängigen Krankheiten vorzubeugen. Gemäss der Weltgesundheitsorganisation (WHO) hängen vier der bedeutendsten Risikofaktoren für nicht übertragbare Krankheiten mit der Ernährung zusammen: geringer Früchte- und Gemüsekonsum, Bluthochdruck, Übergewicht und Adipositas sowie erhöhte Blutcholesterinwerte.

Die Schweizerischen Ernährungsberichte (SEB) des Bundesamtes für Gesundheit (BAG) sind ein wichtiges Instrument für die Ernährungsberichterstattung und erschienen bis anhin im 7-JahresRhythmus. Die Berichte richten sich sowohl an die Fachwelt als auch an die interessierte Bevölkerung. Der 6. SEB, der am 22. Januar 2013 von Bundesrat Alain Berset präsentiert wurde, umfasst rund 300 Seiten mit folgenden Kapiteln:

- Aktuelle Ernährungsempfehlungen

- Ernährungssituation in der Schweiz

- Ernährung und Gesundheit

- Ernährungsmassnahmen zur Förderung der Gesundheit.

\section{Ernährungsempfehlungen}

Die Schweiz verfügt über Ernährungsempfehlungen für verschiedene Alters- und spezifische Bevölkerungsgruppen. Die Empfehlungen basieren auf Grundlagen der WHO und wurden von verschiedenen Fachgesellschaften und Institutionen an schwei-
Im soeben veröffentlichten Sechsten Schweizerischen Ernährungsbericht (6. SEB) werden die aktuelle Ernährungssituation beschrieben und Massnahmen präsentiert, die zu einer Verbesserung der Situation beitragen können. Der Lebensmittelverbrauch (kg pro Kopf) hat sich in den letzten 30 Jahren nicht wesentlich verändert. Der hohe Anteil an übergewichtigen und adipösen Menschen ist eine der grössten Herausforderungen. In der Schweiz gibt es zahlreiche Programme und Projekte zur Förderung einer ausgewogenen Ernährung und ausreichend körperlicher Aktivität auf der Verhaltensund Verhältnisebene. Basierend auf den Erkenntnissen aus dem 6. SEB wurden die sechs Handlungsfelder der Schweizer Ernährungsstrategie 2013-2016 formuliert. Diese legen die Ziele und Prioritäten im Ernährungsbereich fest.

zerische Gegebenheiten angepasst. Es hat sich gezeigt, dass für die Erhaltung der Gesundheit und die Vorbeugung von Krankheiten nicht nur quantitative Empfehlungen (z.B. «5x am Tag Früchte und Gemüse»), sondern auch qualitative (Wahl des Öls aufgrund der Fettsäurenzusammensetzung) von Bedeutung sind. Die Schweizerische Lebensmittelpyramide, die im Dezember 2011 in aktualisierter Form von der Schweizerischen Gesellschaft für Ernährung (SGE) und dem BAG gemeinsam veröffentlicht wurde, zeigt auf, welche Lebensmittel öfter und welche weniger oft konsumiert werden sollten. Viele Menschen in der Schweiz kennen die wichtigsten Ernährungsempfehlungen, setzen diese jedoch aus verschiedenen Gründen nicht oder nur teilweise 


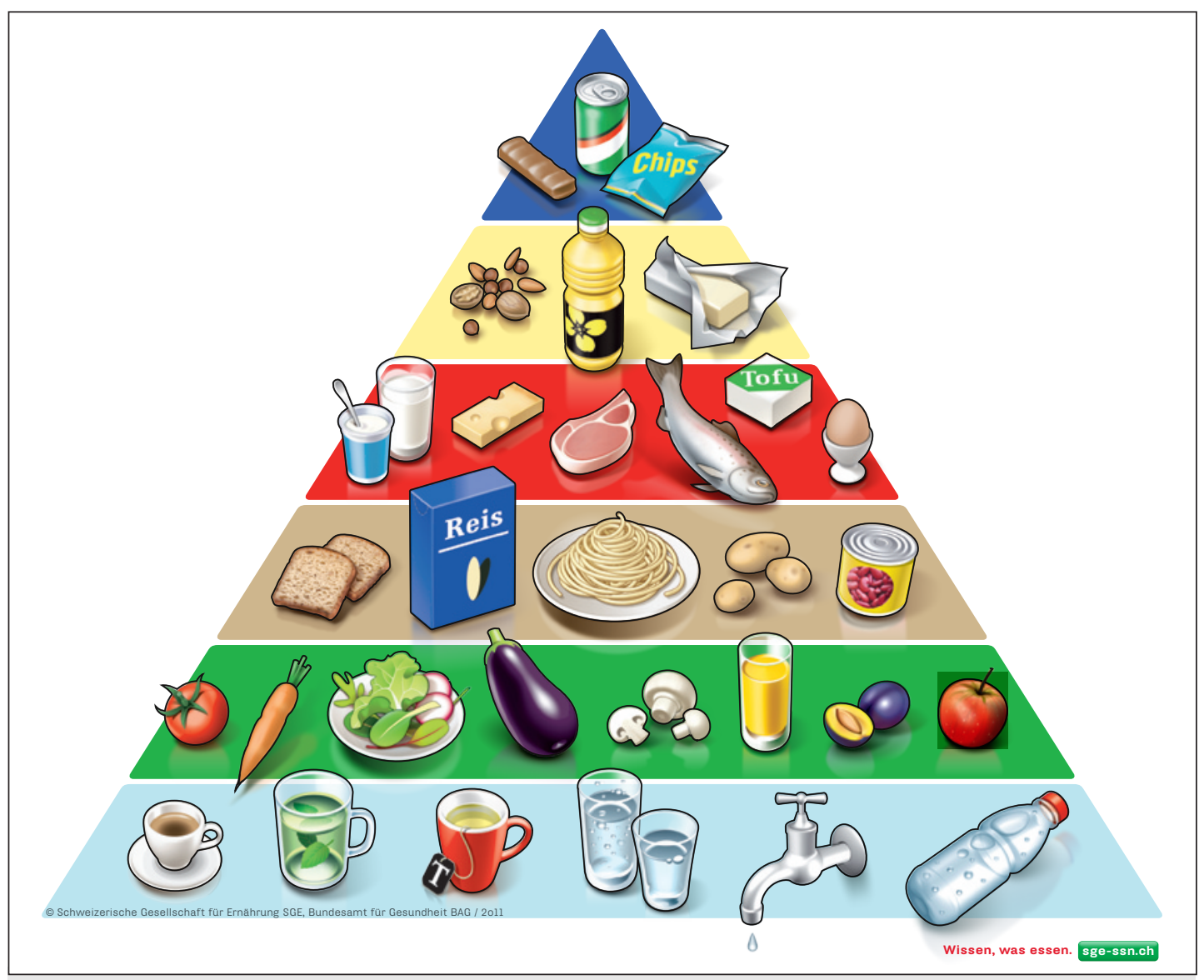

Viele Menschen in der Schweiz kennen die wichtigsten Ernährungsempfehlungen, setzen diese jedoch aus verschiedenen Gründen nicht oder nur teilweise um.

um. Knapp 30\% der Bevölkerung achten auf nichts Bestimmtes bei ihrer Ernährung.

Bei der Formulierung von Ernährungsempfehlungen können Zielkonflikte auftreten. So sind zum Beispiel fettreiche Meerfische eine gute Quelle für langkettige mehrfach ungesättigte Fettsäuren; gleichzeitig können diese Fische aber Schwermetalle enthalten. Um Gefährdungen durch die Empfehlungen auszuschliessen, ist eine sorgfältige NutzenRisiko-Abwägung daher unerlässlich.

Neben den lebensmittelbasierten Empfehlungen wird im 6. SEB auch auf die nährstoffbasierten Empfehlungen eingegangen. Der Fokus liegt dabei auf Natrium bzw. Salz und Vitamin D; bedingt u.a. durch die Salzstrategie des BAG und die Diskussionen über den Bedarf an Vitamin D und die Versorgung in der Schweiz.

\section{Lebensmittelverbrauch und Versorgung mit Nährstoffen}

Die Schweiz verfügt über keine individuellen Lebensmittelverzehrsdaten. Angaben zum Lebensmittelverbrauch, die im 6 . SEB beigezogen wurden, basieren auf der Agrarstatistik und entsprechen den Lebensmittelmengen, die auf dem Schweizer Markt verfügbar sind. Diese Datenlücke, auf die im 6. SEB mehr- fach hingewiesen wird, kann nun mit der vom BAG finanzierten Nationalen Ernährungserhebung geschlossen werden. Der Testdurchlauf der Erhebung startet dieses Jahr.

Der Lebensmittelverbrauch in der Schweiz $(\mathrm{kg}$ pro Kopf) hat sich in den letzten 30 Jahren nicht wesentlich verändert. Auf Basis der Daten zum angenäherten Verzehr scheint die Versorgung mit den meisten Mikronährstoffen ausreichend zu sein. Ungenügend ist hingegen der angenäherte Verzehr an Folsäure und Vitamin D. Nur knapp genügend sind schliesslich die Werte für Eisen, Vitamin B1 und Magnesium. Aufgrund fehlender Daten (z.B. Blutproben) lassen sich keine genaueren Angaben zum Ausmass und der Prävalenz einer allfälligen Unterversorgung mit Mikronährstoffen machen. Es gibt jedoch Hinweise, dass die Versorgung der Bevölkerung oder von Teilen der Bevölkerung mit Vitamin D, Folsäure und Jod unzureichend ist, möglicherweise auch diejenige mit Eisen. Ausreichend ist hingegen die Selenversorgung.

Bereits heute sind ca. ein Fünftel bis ein Viertel aller ins Spital eintretenden Menschen mangelernährt oder weisen ein Risiko für Mangelernährung auf. Diese Problematik dürfte mit dem steigenden Anteil älterer Menschen an Bedeutung gewinnen. 


\section{Ernährung und Gesundheit}

In den vergangenen 30 Jahren stieg die Anzahl der übergewichtigen und adipösen Erwachsenen in der Schweiz kontinuierlich an. Männer sind von Übergewicht (Body-Mass-Index, BMI $=25,0-29,9 \mathrm{~kg} / \mathrm{m}^{2}$ ) deutlich stärker betroffen als Frauen $(37,6 \%$ vs. $20,8 \%$ ); bei Adipositas (BMI $\geq 30 \mathrm{~kg} / \mathrm{m}^{2}$ ) ist der Geschlechtsunterschied deutlich geringer $(8,7 \%$ vs. 7,8\%). Bei den Kindern schwankt der Anteil je nach Alter und Studie zwischen 5-20\% (Übergewicht) bzw. 0,4-6\% (Adipositas). Es gibt Anzeichen, dass sich sowohl bei den Erwachsenen als auch den Kindern die Übergewichts- und Adipositasprävalenz auf hohem Niveau stabilisiert.

Viele ernährungsabhängige Krankheiten wie Bluthochdruck, Diabetes mellitus Typ 2, Herz-Kreislauf-Krankheiten sowie gewisse Krebsformen stehen mit Übergewicht und Adipositas im Zusammenhang. Obwohl Übergewicht und Adipositas im Jahre 2006 direkte und indirekte Kosten von 5,8 Milliarden Franken verursachten, fliessen zurzeit nur gerade 2,3\% aller Ausgaben im Gesundheitssektor in die Gesundheitsförderung und Prävention - der OECD-Durchschnitt liegt bei 3,1\%. und Gemüsekonsums bei Kindern und die Optimierung der Zusammensetzung von Angeboten in der Ausser-Haus-Verpflegung. Verantwortlich für eine erfolgreiche Umsetzung der Massnahmen sind u.a. Behörden aus verschiedenen Politikbereichen, Schulen/Bildungseinrichtungen, Nichtregierungsorganisationen sowie die Privatwirtschaft (z.B. Lebensmittelindustrie, Gastronomie).

Die Schweizer Ernährungsstrategie 2013-2016 Die Situationsanalyse, die mit dem 6. SEB gemacht wurde, ermöglicht es, Problemfelder im Ernährungsbereich zu identifizieren. Daraus können Handlungsfelder abgeleitet werden, innerhalb derer konkrete Massnahmen zur Verbesserung der Ernährungs- und Gesundheitssituation in der Schweiz ergriffen werden sollten. Das BAG hat daher die Veröffentlichung des 6 . SEB zum Anlass genommen, die Ernährungspolicy aus dem Jahre 2001 zu überarbeiten und zeitgleich zum 6. SEB die Schweizer Ernährungsstrategie 2013-2016 zu publizieren. Die auf Departementsebene verabschiedete Schweizer Ernährungsstrategie legt die Ziele und Prioritäten im Ernährungsbereich fest und bildet gleichzeitig für

\section{Bereits heute sind ca. ein Fünftel bis ein Viertel aller ins Spital eintretenden Menschen mangelernährt.}

\section{Laufende Aktivitäten, Zielgruppen und Handlungsempfehlungen}

In der Schweiz laufen zahlreiche Programme und Projekte zur Förderung einer ausgewogenen Ernährung und ausreichend körperlicher Aktivität. Als erfolgversprechend haben sich Massnahmen erwiesen, die das Umfeld bzw. die Lebenswelten der Zielgruppe berücksichtigen und sowohl auf der Verhaltens- als auch auf der Verhältnisebene ansetzen. Als besondere Zielgruppen zukünftiger Ernährungsinterventionen sollten Männer, junge Menschen und sozio-ökonomisch benachteiligte Bevölkerungsgruppen besonders angesprochen werden. Dabei sollten Massnahmen auf der Verhältnisebene im Vordergrund stehen.

Im 6. SEB sind Handlungsempfehlungen und dringliche Massnahmen für die Schweiz formuliert worden. Zu Letzteren gehören z. B. die Verbesserung der Datenlage, die Stärkung der Gesundheits- und Ernährungskompetenzen, die Förderung des Früchte- die verschiedenen Akteure eine Grundlage für die Erarbeitung eigener Aktionspläne und Massnahmen. Die Schweizer Ernährungsstrategie bildet zudem die zentrale Grundlage für das Nationale Programm Ernährung und Bewegung NPEB, das im Sommer 2012 vom Bundesrat bis Ende 2016 verlängert wurde. Die erfolgreiche Umsetzung erfordert das Zusammenspiel von Interventionen auf der Verhaltens- und Verhältnisebene sowie die Zusammenarbeit diverser Akteure nicht nur aus dem Ernährungs- und Gesundheitsbereich, sondern auch aus anderen Bereichen wie der Wirtschaft, der Bildung und der Landwirtschaft.

Der 6. SEB und die Schweizer Ernährungsstrategie 2013-2016 (Broschüre) sind in elektronischer Form kostenlos in deutscher, französischer und italienischer Sprache verfügbar:

- www.ernaehrungsbericht.ch

- www.rapportsurlanutrition.ch

- www.rapportosullalimentazione.ch 